\title{
The Effect of Chemical and Non-Chemical Control Methods on Weeds and Yield in Potato (Solanum tuberosum L.) Cultivation under Potato Based Organic Cropping System
}

\author{
Varsha Gupta*, Ekta Joshi, Deep Singh Sasode Lakhan Singh, \\ B.S. Kasana and Y.K. Singh \\ Rajmata Vijayaraje Scindia Krishi Vishwa Vidhyalaya, Gwalior, \\ Madhya Pradesh 474002, India \\ *Corresponding author
}

\begin{tabular}{l} 
K e y w or d s \\
Potato, Hand \\
weeding, Weeds, \\
Non-chemical, \\
Plastic mulch, \\
Straw mulch, Yield \\
Article Info \\
$\begin{array}{l}\text { Accepted: } \\
\text { 20 June } 2019 \\
\text { Available Online: } \\
10 \text { July } 2019\end{array}$ \\
\hline
\end{tabular}

\section{A B S T R A C T}

Potato is one of the most important commercial vegetable crops in India and on an average this crop is affected by weeds damage which should be prevented by various control methods. Cultural operations and mulches are non-chemical methods of weed control in sustainable agricultural systems. The aim of present study was to investigate the effect of chemical and non-chemical control methods on weeds and yield of potato crop. Field experiments were conducted during rabi 2016-17 and 2017-18 at Research Farm College of Agriculture, RVSKVV, Gwalior in a randomized block design (RBD) with three replications. The treatments were as follows: 1) white plastic mulch, 2) black plastic mulch, 3) straw mulching after $5 \mathrm{DAP}$ as $5 \mathrm{t} / \mathrm{ha}, 4)$ one hand weeding at $20 \mathrm{DAP}+$ straw mulching at 25 DAP, 5) two hand weeding at $20 \& 40$ DAP, 6) one hand hoeing at 20 DAP, 7) one hand hoeing at $20 \mathrm{DAP}+$ one hand weeding at $40 \mathrm{DAP}, 8)$ Recommended herbicide (metribuzin $500 \mathrm{~g} / \mathrm{ha}$ ), 9) Recommended herbicide (metribuzin $500 \mathrm{~g} / \mathrm{ha}$ ) + one hand weeding at 40 DAP and 10) Weedy check. Based on results, all treatments were affected by weed control methods. At 60 DAP the highest weeds density was counted as 567.83 plants $/ \mathrm{m}^{2}$ of weedy check treatment. The two hand weeding at $20 \& 40$ DAP deceased weed density $86 \%$ as compared to weedy check. The hoeing at 20 DAP with one hand weeding at 40 DAP and straw mulching with one hand weeding were also deceased weeds density around $75 \%$ comparing to weedy check. However, at harvest stage weed biomass in the treatments of white and black plastic mulch compared to the weed infested treatment had the highest weeds biomass around $46 \%$ in both. The lowest weed biomass was observed in two hand weeding at 20 and 40 DAP by $19.22 \%$ after the weedy check. It can be concluded that, the two hand weeding at 20 and 40 DAP and straw mulching with one hand weeding can provide more proper control of weeds compared to the use of herbicides and the use of straw mulches reduces the use of herbicides. 


\section{Introduction}

Potato is one of the most important commercial vegetable crops widely grown in India. There are several constraints in potato production, but weeds often pose a serious problem. Potato crop have robust growing and quick spreading nature but it turns as a weak competitor with weeds. Weeds not only compete with crop plants for nutrients, space, sunlight and soil moisture but also serve as an alternative hosts for several insect pest and diseases. Liberal use of manures and fertilizers, spacing and frequent irrigations provide favorable conditions for an early start of weeds well before the emergence of potato plant. Under irrigated conditions it was observed that the most critical period of cropweed competition in potato is 20 to 66 days after emergence when the crop must be kept free from weeds (Monteiro et al., 2011). (Singh and Bhan (1999) reported that the presence of weeds throughout the growing season caused $62 \%$ reduction in tuber yield. The yield reduction due to weeds in potato is estimated to be as high as 10 to 80 per cent (Lal and Gupta, 1984). Therefore, weed control in the initial stages plays an important role in maximizing the tuber production. Farmers in this region usually grow potato without having proper knowledge on use of herbicide.

In most potato producer countries in the world with different climatic conditions, weed control is carried out by agricultural, mechanical and chemical methods or their combinations in potato fields (Hutchinson et al., 2011; Kunz et al., 2015; Weber et al., 2016). Metribuzin is mostly used in potato fields both before planting and pre-emergence to control narrow and broad leaved weeds (Zand et al., 2007; Zaki et al., 2014). Chemical weed control appears to hold a great promise in dealing with effective, timely and economic weed suppression. Besides beneficial effects of chemical weed control it also creates environmental hazards and develops herbicide resistance \& weed shift. Weed interference with the crop reduces marketable yield by decreasing number and size of potato tuber (Ahuja et al., 1999; Singh and Bhan, 1999). Weeds may also hinder mechanical harvesting (Pandey, 2000; Wall and Friesen, 1990). The use of tillage or cultivator along with herbicide, controls the weeds effectively and it is used as one of the major methods of weed control in potato crop (Mohammaddoust et al., 2011).

In these days, mulches are used as an organic method for weed management. The application of plant mulch has a great importance to expand sustainable agriculture systems. The need to increase farm productivity and profitability is a factor that stimulates the development of methods based on the ecological management of weeds. If the better ecological process is used in weed management, agricultural income will be increased by reducing the inputs cost and helping farmers to provide market friendly agricultural products (Dejam et al., 2010; Jafari et al., 2013; Najafi et al., 2016). Specifically, when using non-chemical methods of weed control, the competition of weeds with crop plants will reduce and as a result the use of other methods (as chemical control) to control weeds will be reduced. Keeping all these things in view the investigation was planned to find out suitable and economically viable weed management practices for potato.

\section{Materials and Methods}

\section{Study location}

Field experiments were conducted during rabi 2016-17 and 2017-18 at research farm (Fig. 1) College of Agriculture, RVSKVV, Gwalior, Madhya Pradesh, India with an altitude of 
$411.98 \mathrm{~m}$ from sea level, a longitude of $79^{\circ}$ $54^{\prime} \mathrm{E}$ and a latitude of $23^{\circ} 10^{\prime} \mathrm{N}$ in the central part of Madhya Pradesh. The mean temperature varies between $5^{0} \mathrm{C}$ in DecemberJanuary to $46^{\circ} \mathrm{C}$ in May-June. The mean rainfall is $751 \mathrm{~mm}$ which is mostly received in between July to September (Fig. 2). The soil was sandy clay loam in texture, low in available nitrogen $(237 \mathrm{~kg} / \mathrm{ha})$, medium in phosphorus (19.7 kg/ha) and potassium (277.1 $\mathrm{kg} / \mathrm{ha}$ ). This is the major area of potato cultivation and many researches are being made in this area.

\section{Experimental design and treatments}

The 10 treatments were replicated thrice in RBD are 1) The application of white plastic mulch, covering the space between the rows with plastic sheets with the thickness of 50 microns and then potato planting was done with making a whole in the plastic sheet immediately. 2) The application of black plastic mulch was similar to that of treatment one. 3)Wheat straw mulch with the amount of $5 \mathrm{t} / \mathrm{ha}$ and thickness of $15 \mathrm{~cm}$ after 5 days of planting (DAP) 4) One hand weeding was done after 20 days of planting (DAP) + wheat straw mulch was applied after 5 days of hand weeding (25 DAP). 5) Two hand weeding at $20 \& 40$ DAP. 6) One hand hoeing was done after 20 days of planting potato. 7).

One hand hoeing at 20 DAP + one hand weeding at 40 DAP. 8) Spraying metribuzin herbicide on the soil between the rows of potato plants with the amount of $500 \mathrm{~g} / \mathrm{ha}$ (with the formulation of $70 \%$ WP by the flat fan nozzle and the constant speed and pressure of sprayer in the treatment spraying; thus, the metribuzin was mixed with soil at the depth of $0-10 \mathrm{~cm}$ ) immediately after potato planting. 9) Application of metribuzin herbicide such as the $\mathrm{T}_{8}$ treatment with one hand weeding at 40 DAP. 10) Weedy check or no weed removal in the entire growing season in both season.

\section{Land preparation and planting}

Field was prepared to a fine tilth by one deep ploughing followed by two cross disc harrowing and planking. Variety "Kufri Sindoori" $30 \mathrm{q} /$ ha with the average weight of $30 \mathrm{~g}$ was planted in the plots $\left(5 \times 3.6 \mathrm{~m}^{2}\right)$ with spacing of $60 \mathrm{~cm} \times 20 \mathrm{~cm}$ after harvesting of greengram. Due to organic cropping system, seed treatment was done with phosphate solubilising bacteria (PSB) $5 \mathrm{ml} / \mathrm{kg}$ by dipping tubers for 30 minutes to control soil and tuber born diseases, increases the root system and to provide the strength to the plant. The potatoes were planted in the month of November in the both season rabi 2016 and 2017. A full dose of FYM (10 t/ha) and half dose of vermicompost 5 t/ha were applied before 15 days of planting during field preparation. The rest half dose of vermicompost (5 t/ha) with gram flour (besan) $5 \mathrm{~kg} / \mathrm{ha}$ was applied before planting in the field. Neem cake $250 \mathrm{~kg} / \mathrm{ha}$ was applied in the soil to control termites. Foliar spray of panchagavya (3\% solution) at 10 days interval was applied 5 times entire the crop growth period in the field. During first irrigation PSB+KSB+Azotobacter $\quad(100 \mathrm{ml}$ each/ha) was also applied to provide enzymes, vitamins and hormones to the plants. White and black plastic mulches were placed in the field during the sowing time. The herbicide metribuzin $500 \mathrm{~g} / \mathrm{ha}$ was sprayed as preemergence in $T_{8}$ and $T_{9}$ plot. The crop was harvested in the month of February during both the season.

\section{Potato and weed samples}

Weeds were sampled according to species and were counted. Weed sampling were performed in each plot with dimensions of 1.0 $x 1.0 \mathrm{~cm}^{2}$ of the soil surface and were shifted to the laboratory. After counting the weeds, separate each species and keep in special bags and placed at oven for 72 hours at $75^{\circ} \mathrm{C}$. After drying the samples, it was weighed by 
balance with precision of $0.01 \mathrm{~g}$. For data uniformity, transform value $\sqrt{x+0.5}$ was used related to weed density. Yield of tuber was recorded after completion of the growing season and ripening potato tuber, the middle rows of plants (excluding two border rows) were collected for estimation of yield per plot and convert it in to the $\mathrm{t} / \mathrm{ha}$. The Weed control efficiency (WCE\%) was also worked out at 30 and 60 days after planting (DAP) and at harvest stage for different weed management practices.

\section{Results and Discussion}

\section{Weed flora}

The major weed flora of experimental site during both the season were Cyperus rotundus (38.27\%), Phalaris minor (18.45\%), Spergula arvensis (2.17\%), Polypogon monspeliensis (2.26\%), Rumex dentatus (1.50\%), Avena fatua (0.62\%), Hordium spontaneum (2.69\%), and Cynodon dactylon (1.48\%) as grasses, Medicago polymorpha (3.03\%), Chenopodium album (24.55\%), Convolvulus arvensis (1.38\%) and Anagallis arvensis (3.58\%) as major broad-leaved weeds (BLW's). While Cynodon dactylon and Rumex dentatus have not seen in 2017-18 in the field of potato (Table 1).

Some weeds i.e. Avena fatua, Medicago polymorpha, Polypogon monspeliensis and Anagallis arvensis were emerged only at 60 DAP in potato field. The sedges Cyperus rotundus was most dominating weed among all during both the season in potato field.

$\mathrm{C}_{3}$ grasses have generally more nutritional quality than $\mathrm{C}_{4}$ grasses at elevated $\mathrm{CO}_{2}$ concentrations, having higher levels of protein, nonstructural carbohydrates and water, but lower levels of fiber and toughness than $\mathrm{C}_{4}$ grasses (Raymond et al., 2004).

\section{Effect on weeds}

Weed population and dry weight of weeds were significantly affected by all weed management practices. In the initial stage of crop growth up to 30 days of planting the minimum weed density was found in two hand weeding treatment followed by one hand weeding with straw mulc. But after 60 days of planting, among non chemical methods of weed control the density of weeds was effectively controlled by two hand weeding followed by one hoeing with one hand weeding at 40 DAP and it was very close to straw mulch with one hand weeding. Sedges were also controlled by two hand weeding effectively (Table 2).

Dry weight of weeds was significantly affected by different treatments. After 30 days of planting the minimum dry weight production of weeds was found under one hand weeding with Straw mulch $5 \mathrm{t} / \mathrm{ha}$ which was closely followed by two hand weeding treatment. The minimum dry weight at 60 DAP was found with two hand weeding treatment followed by one hand weeding with straw mulching. After 30 days of planting the maximum weed control efficiency $(73.46 \%)$ was recorded in one hand weeding with straw mulch followed by two hand weeding $(72.45 \%)$ and minimum weed control efficiency was found where white plastic sheet was used as compared to black plastic mulch.

The light passes through white plastic mulch is likely to stimulate the germination of weeds but because of the low temperature at an experimental site could not prevent weeds germination and the result, may be stimulated weed species and causes of weed germination. Similar results were also reported by Azadbakht et al., (2017), Majd et al., (2014), Sand Singh (1974) and Sood and Sharma (1985) in this regard. 


\section{Effect on yield}

In present experiment all treatments have significantly affected the yield of potato crop. In the treatments where mulching was done with straw, yield was more than 22 t ha ${ }^{-1}\left(T_{3}\right.$ and $\left.T_{4}\right)$. In $T_{1}$ where white plastic mulch was applied, yield was 18.70 $\mathrm{t} \mathrm{ha}^{-1}$ (Table 4). However, highest yield $(27.32 \mathrm{t} / \mathrm{ha})$ was recorded in the treatment where two hand weeding was done then one hand weeding with straw mulch $(25.39 \mathrm{t} / \mathrm{ha})$ and straw mulch alone (22.96 t/ha) (Table 3). Lowest yield (14.20 t/ha) was obtained in $\mathrm{T}_{10}$ Weedy check treatment. Overall increase in yield was $92 \%$ in $\mathrm{T}_{5}, 79 \%$ in $\mathrm{T}_{4}, 62 \%$ in $\mathrm{T}_{3}, 58 \%$ in $\mathrm{T}_{9}, 54 \%$ in $\mathrm{T}_{8}$ and $43 \%$ in $\mathrm{T}_{2}$ over $\mathrm{T}_{10}$ weedy check (Table 4). Mulching with straw was superior to plastic in terms of $\mathrm{B}: \mathrm{C}$ ratios. These results are supported the findings of Khalak and Kumaraswamy (1993) and Masud Mahmood (2002). Highest root temperature caused by black plastic $\left(>31{ }^{\circ} \mathrm{C}\right)$ depressed nitrogen metabolism as well as yield (Momirovic et al., 1997 and Ruiz et al., 1999).

\section{Economics}

Application of two hand weeding at 20 and 40 DAP fetched significantly highest net returns (Rs.318346/ha) followed by one hand weeding at 20 DAP with straw mulching 5 t/ha at 25 DAP (Rs. 286549/ha) and straw mulching $5 \mathrm{t} / \mathrm{ha}$ at 5 DAP (Rs.254241/ha). Similarly, the maximum B:C ratio of 3.40 was also obtained with two hand weeding at 20 and 40 DAP followed by one hand weeding at 20 DAP with straw mulching 5 t/ha at 25 DAP (2.94) which was at par with straw mulching 5 t/ha at 5 DAP (2.84), The minimum net returns Rs. 129600/ha was recorded in weedy check with $\mathrm{B}: \mathrm{C}$ ratio of 1.49 (Table 4). In terms of economic benefit straw mulch was beneficial over polythene mulch as the $\mathrm{B}: \mathrm{C}$ ratio was higher with straw mulches when compared to polythene. Further the residues of straw added to the organic matter status apart from improving physico-chemical properties of soil.

Table.1 The biological and photosynthetic traits of weed species observed in the sampling units of potato field

\begin{tabular}{|c|c|c|c|c|c|}
\hline S.No & Common name & Scientific name & Family & Life cycle & $\begin{array}{l}\text { Photosynthetic } \\
\text { cycle }\end{array}$ \\
\hline \multicolumn{6}{|c|}{ NLWs } \\
\hline 1. & Gehunsa & Phalaris minor $L$. & Poaceae & Annual & $\mathrm{C}_{4}$ \\
\hline 2. & Corn spurry & Spergula arvensis $L$. & Caryophyllaceae & Annual & $\mathrm{C}_{4}$ \\
\hline 3. & Bermuda Grass & Cynodon dactylon $L$. & Poaceae & Perennial & $\mathrm{C}_{4}$ \\
\hline 4. & Toothed dock & Rumax dentatus $L$. & Polygonaceae & Annual/Biennial & $\mathrm{C}_{3}$ \\
\hline 5. & Wild barley & Hordium spontaneum & Poaceae & Annual & $\mathrm{C}_{3}$ \\
\hline 6. & Rabbits foot grass & Polypogon monspeliensis & Poaceae & Annual & $\mathrm{C}_{3}$ \\
\hline 7. & Wild oat & Avena fatua $L$. & Poaceae & Annual & $\mathrm{C}_{3}$ \\
\hline \multicolumn{6}{|l|}{ BLWs } \\
\hline 8. & Lamb's quarters & Chenopodium album $L$. & Chenopodiaceae & Annual & $\mathrm{C}_{3}$ \\
\hline 9. & Bindweed & Convolvulus arvensis $L$. & Convolvulaceae & Perennial & $\mathrm{C}_{3}$ \\
\hline 10. & Red scarlet & Anagallis arvensis $L$. & Primulaceae & Annual & $\mathrm{C}_{3}$ \\
\hline 11. & Bur clover & Medicago polymorpha & $\underline{\text { Fabaceae }}$ & Annual & $\mathrm{C}_{3}$ \\
\hline \multicolumn{6}{|c|}{ Sedges } \\
\hline 12. & Nut sedge & Cyperus rotundus & Cyperaceae & Perennial & $\mathrm{C}_{4}$ \\
\hline
\end{tabular}

NLWs=Narrow Leaved Weeds, BLWs $=$ Broad Leaved Weeds 
Table.2 Effect of different weed management practices on weed density/m2 during 2016-17 and 2017-18 (pooled data)

\begin{tabular}{|c|c|c|c|c|c|c|c|c|c|c|}
\hline \multicolumn{2}{|r|}{ Treatments } & \multicolumn{4}{|c|}{30 DAP } & \multicolumn{5}{|c|}{60 DAP } \\
\hline & & Narrow & Broad & Sedges & Total & Narrow & Broad & Sedges & Total & $\begin{array}{c}\% \text { weed } \\
\text { density } \\
\text { decreased }\end{array}$ \\
\hline $\mathbf{T}_{1}$ & White plastic mulch & $\begin{array}{c}7.30 \\
(57.33)\end{array}$ & $\begin{array}{c}7.34 \\
(71.33)\end{array}$ & $\begin{array}{c}9.00 \\
(80.67)\end{array}$ & $\begin{array}{c}14.08 \\
(209.33)\end{array}$ & $\begin{array}{c}8.57 \\
(78.00)\end{array}$ & $\begin{array}{c}11.36 \\
(136.00)\end{array}$ & $\begin{array}{c}8.90 \\
(80.67)\end{array}$ & $\begin{array}{c}16.78 \\
(294.67)\end{array}$ & 48.11 \\
\hline $\mathbf{T}_{2}$ & Black plastic mulch & $\begin{array}{c}6.98 \\
(51.00)\end{array}$ & $\begin{array}{c}7.99 \\
(74.67)\end{array}$ & $\begin{array}{c}7.00 \\
(49.33)\end{array}$ & $\begin{array}{c}12.85 \\
(175.00)\end{array}$ & $\begin{array}{c}7.49 \\
(64.67)\end{array}$ & $\begin{array}{c}9.43 \\
(100.00)\end{array}$ & $\begin{array}{c}8.60 \\
(75.33)\end{array}$ & $\begin{array}{c}14.86 \\
(240.00)\end{array}$ & 57.73 \\
\hline $\mathbf{T}_{3}$ & Straw mulching $5 \mathrm{t} / \mathrm{ha}$ at $5 \mathrm{DAP}$ & $\begin{array}{c}6.80 \\
(51.67)\end{array}$ & $\begin{array}{c}7.16 \\
(60.67)\end{array}$ & $\begin{array}{c}8.42 \\
(72.00)\end{array}$ & $\begin{array}{c}13.43 \\
(184.33)\end{array}$ & $\begin{array}{c}7.38 \\
(56.00)\end{array}$ & $\begin{array}{c}10.22 \\
(108.67)\end{array}$ & $\begin{array}{c}7.43 \\
(55.33)\end{array}$ & $\begin{array}{c}14.63 \\
(220.00)\end{array}$ & 61.26 \\
\hline $\mathbf{T}_{4}$ & $\begin{array}{l}\text { One } \mathrm{HW} \text { at } 20 \mathrm{DAP}+\mathrm{Straw} \\
\text { mulching } 5 \text { t/ha at } 25 \mathrm{DAP}\end{array}$ & $\begin{array}{c}4.95 \\
(26.17)\end{array}$ & $\begin{array}{c}6.14 \\
(45.67)\end{array}$ & $\begin{array}{c}7.53 \\
(58.67)\end{array}$ & $\begin{array}{c}10.95 \\
(130.50)\end{array}$ & $\begin{array}{c}5.72 \\
(32.50)\end{array}$ & $\begin{array}{c}7.50 \\
(58.17)\end{array}$ & $\begin{array}{c}7.19 \\
(51.33)\end{array}$ & $\begin{array}{c}11.89 \\
(142.00)\end{array}$ & 74.99 \\
\hline $\mathbf{T}_{5}$ & $\begin{array}{l}\text { Two hand weeding at } 20 \& 40 \\
\text { DAP }\end{array}$ & $\begin{array}{c}5.37 \\
(35.33)\end{array}$ & $\begin{array}{c}5.80 \\
(44.67)\end{array}$ & $\begin{array}{c}4.92 \\
(27.17)\end{array}$ & $\begin{array}{c}9.29 \\
(107.17)\end{array}$ & $\begin{array}{c}4.41 \\
(19.50)\end{array}$ & $\begin{array}{c}5.71 \\
(35.00)\end{array}$ & $\begin{array}{c}4.81 \\
(22.83)\end{array}$ & $\begin{array}{c}8.65 \\
(77.33)\end{array}$ & 86.38 \\
\hline $\mathbf{T}_{6}$ & One hand hoeing at $20 \mathrm{DAP}$ & $\begin{array}{c}6.28 \\
(44.67)\end{array}$ & $\begin{array}{c}8.48 \\
(74.50)\end{array}$ & $\begin{array}{c}7.81 \\
(60.67)\end{array}$ & $\begin{array}{c}13.22 \\
(179.83)\end{array}$ & $\begin{array}{c}6.92 \\
(48.00)\end{array}$ & $\begin{array}{c}9.05 \\
(81.67)\end{array}$ & $\begin{array}{c}9.39 \\
(89.33)\end{array}$ & $\begin{array}{c}14.81 \\
(219.00)\end{array}$ & 61.43 \\
\hline $\mathbf{T}_{7}$ & $\begin{array}{l}\text { Hoeing at } 20 \mathrm{DAP}+\text { hand } \\
\text { weeding at } 40 \mathrm{DAP}\end{array}$ & $\begin{array}{c}7.53 \\
(60.00)\end{array}$ & $\begin{array}{c}8.85 \\
(78.00)\end{array}$ & $\begin{array}{c}9.52 \\
(99.33)\end{array}$ & $\begin{array}{c}15.38 \\
(237.33)\end{array}$ & $\begin{array}{c}6.50 \\
(45.83)\end{array}$ & $\begin{array}{c}6.70 \\
(48.67)\end{array}$ & $\begin{array}{c}6.20 \\
(42.67)\end{array}$ & $\begin{array}{c}11.17 \\
(137.17)\end{array}$ & 75.84 \\
\hline $\mathbf{T}_{8}$ & $\begin{array}{l}\text { Recommended herbicide } \\
\text { (metribuzin } 0.5 \mathrm{~kg} / \mathrm{ha} \text { ) }\end{array}$ & $\begin{array}{c}7.48 \\
(57.33)\end{array}$ & $\begin{array}{c}3.56 \\
(13.17)\end{array}$ & $\begin{array}{c}12.38 \\
(160.67)\end{array}$ & $\begin{array}{c}15.16 \\
(231.17)\end{array}$ & $\begin{array}{c}8.30 \\
(70.00)\end{array}$ & $\begin{array}{c}6.08 \\
(39.17)\end{array}$ & $\begin{array}{c}11.15 \\
(127.33)\end{array}$ & $\begin{array}{c}15.17 \\
(236.50)\end{array}$ & 58.35 \\
\hline $\mathbf{T}_{9}$ & $\begin{array}{l}\text { Recommended herbicide }+ \text { one } \\
\text { HW at } 40 \text { DAP }\end{array}$ & $\begin{array}{c}5.65 \\
(42.33)\end{array}$ & $\begin{array}{c}2.46 \\
(7.67)\end{array}$ & $\begin{array}{c}10.73 \\
(116.67)\end{array}$ & $\begin{array}{c}12.89 \\
(166.67)\end{array}$ & $\begin{array}{c}6.84 \\
(48.83)\end{array}$ & $\begin{array}{c}4.73 \\
(23.17)\end{array}$ & $\begin{array}{c}9.84 \\
(100.00)\end{array}$ & $\begin{array}{c}12.85 \\
(172.00)\end{array}$ & 69.71 \\
\hline $\mathbf{T}_{10}$ & Weedy check & $\begin{array}{c}11.82 \\
(153.50)\end{array}$ & $\begin{array}{c}12.72 \\
(191.00)\end{array}$ & $\begin{array}{c}16.50 \\
(272.00)\end{array}$ & $\begin{array}{c}24.43 \\
(616.50)\end{array}$ & $\begin{array}{c}11.97 \\
(147.00)\end{array}$ & $\begin{array}{c}14.09 \\
(210.17)\end{array}$ & $\begin{array}{c}14.51 \\
(210.67)\end{array}$ & $\begin{array}{c}23.58 \\
(567.83)\end{array}$ & - \\
\hline & $\operatorname{SEm}( \pm)$ & 0.320 & 0.364 & 0.501 & 0.435 & 0.389 & 0.339 & 0.372 & 0.346 & - \\
\hline & $(\mathrm{LSD}=\mathbf{0 . 0 5})$ & 0.938 & 1.067 & 1.467 & 1.274 & 1.139 & 0.992 & 1.089 & 1.012 & - \\
\hline & $\mathrm{CV} \%$ & 7.908 & 8.950 & 9.250 & 5.319 & 9.091 & 6.913 & 7.324 & 4.149 & - \\
\hline
\end{tabular}


Table.3 Effect of different weed management practices on weed biomass g/m2 during 2016-17 and 2017-18 (pooled data)

\begin{tabular}{|c|c|c|c|c|c|c|c|c|c|c|c|c|c|c|c|}
\hline \multicolumn{2}{|r|}{ Treatments } & \multicolumn{5}{|c|}{30 DAP } & \multicolumn{6}{|c|}{60 DAP } & \multicolumn{3}{|c|}{ At harvest } \\
\hline & & Narrow & Broad & Sedges & Total & WCE\% & Narrow & Broad & Sedges & Total & WCE\% & $\begin{array}{c}\text { Weed } \\
\text { biomass } \\
(\%)\end{array}$ & $\begin{array}{c}\text { Weed } \\
\text { biomass } \\
\left(\mathrm{g} / \mathrm{m}^{2}\right)\end{array}$ & WCE\% & $\begin{array}{l}\text { Weed } \\
\text { biomass } \\
(\%)\end{array}$ \\
\hline $\mathbf{T}_{1}$ & White plastic mulch & 21.87 & 8.51 & 12.60 & 42.98 & 52.81 & 23.50 & 22.37 & 4.03 & 49.91 & 45.46 & 54.54 & 2.46 & 54.17 & 45.83 \\
\hline $\mathbf{T}_{2}$ & Black plastic mulch & 18.13 & 8.19 & 9.49 & 35.81 & 60.68 & 18.17 & 16.64 & 3.77 & 38.57 & 57.84 & 42.16 & 2.50 & 53.39 & 46.61 \\
\hline $\mathbf{T}_{3}$ & $\begin{array}{l}\text { Straw mulching } 5 \\
\text { t/ha at } 5 \text { DAP }\end{array}$ & 18.23 & 6.78 & 8.68 & 33.69 & 63.01 & 17.60 & 17.82 & 2.77 & 38.19 & 58.27 & 41.73 & 1.09 & 79.70 & 20.30 \\
\hline $\mathbf{T}_{4}$ & $\begin{array}{l}\text { One } \mathrm{HW} \text { at } 20 \mathrm{DAP} \\
+ \text { Straw mulching } 5 \\
\text { t/ha at } 25 \mathrm{DAP}\end{array}$ & 10.83 & 5.37 & 7.97 & 24.17 & 73.46 & 9.66 & 9.54 & 2.13 & 21.33 & 76.69 & 23.31 & 0.73 & 86.44 & 13.56 \\
\hline $\mathbf{T}_{5}$ & $\begin{array}{l}\text { Two hand weeding at } \\
20 \& 40 \text { DAP }\end{array}$ & 12.75 & 5.37 & 6.97 & 25.10 & 72.45 & 8.04 & 9.39 & 2.14 & 19.58 & 78.61 & 21.39 & 1.03 & 80.78 & 19.22 \\
\hline $\mathbf{T}_{6}$ & $\begin{array}{l}\text { One hand hoeing at } \\
20 \text { DAP }\end{array}$ & 17.67 & 6.96 & 13.78 & 38.41 & 57.83 & 16.58 & 13.00 & 4.47 & 34.05 & 62.79 & 37.21 & 2.29 & 57.21 & 42.79 \\
\hline $\mathbf{T}_{7}$ & $\begin{array}{l}\text { Hoeing at } 20 \mathrm{DAP}+ \\
\text { hand weeding at } 40 \\
\text { DAP }\end{array}$ & 17.73 & 5.15 & 13.33 & 36.21 & 60.24 & 13.46 & 8.06 & 2.13 & 23.65 & 74.15 & 25.85 & 1.22 & 77.33 & 22.67 \\
\hline $\mathbf{T}_{8}$ & $\begin{array}{l}\text { Recommended } \\
\text { herbicide (metribuzin } \\
0.5 \mathrm{~kg} / \mathrm{ha} \text { ) }\end{array}$ & 15.80 & 1.87 & 15.37 & 33.03 & 63.73 & 22.72 & 5.75 & 6.37 & 34.83 & 61.93 & 38.07 & 1.34 & 74.94 & 25.06 \\
\hline $\mathbf{T}_{9}$ & $\begin{array}{l}\text { Recommended } \\
\text { herbicide + one HW } \\
\text { at } 40 \text { DAP }\end{array}$ & 15.75 & 1.39 & 10.98 & 28.12 & 69.12 & 14.60 & 3.81 & 5.00 & 23.41 & 74.41 & 25.59 & 1.65 & 69.15 & 30.85 \\
\hline$T_{10}$ & Weedy check & 47.87 & 20.97 & 22.25 & 91.08 & & 46.23 & 34.74 & 10.53 & 91.50 & & & 5.36 & & \\
\hline & $\operatorname{SEm}( \pm)$ & 1.61 & 0.43 & 0.85 & 2.08 & & 1.35 & 1.27 & 0.36 & 1.79 & & & 0.19 & & \\
\hline & $(\mathrm{LSD}=\mathbf{0 . 0 5})$ & 4.70 & 1.27 & 2.49 & 6.08 & & 3.97 & 3.72 & 1.06 & 5.25 & & & 0.54 & & \\
\hline & CV\% & 14.16 & 10.67 & 12.15 & 9.25 & & 12.32 & 15.60 & 14.46 & 8.28 & & & 16.33 & & \\
\hline
\end{tabular}


Table.4 Effect of different weed management practices on yield and economics of potato at harvest during 2016-17 and 2017-18 (pooled data)

\begin{tabular}{|c|c|c|c|c|c|c|c|c|}
\hline \multicolumn{2}{|c|}{ Treatments } & \multirow{2}{*}{$\begin{array}{c}\begin{array}{c}\text { Tuber } \\
\text { yield t/ha }\end{array} \\
18.69\end{array}$} & \multirow{2}{*}{$\begin{array}{c}\begin{array}{c}\text { Difference } \\
\text { t/ha }\end{array} \\
4.49 \\
\end{array}$} & \multirow{2}{*}{$\begin{array}{c}\begin{array}{c}\% \text { yield } \\
\text { increased }\end{array} \\
31.67\end{array}$} & \multirow{2}{*}{$\begin{array}{c}\% \text { yield } \\
\text { reduced }\end{array}$} & \multirow{2}{*}{$\begin{array}{c}\begin{array}{c}\text { Gross } \\
\text { returns }\end{array} \\
280415\end{array}$} & \multirow{2}{*}{$\begin{array}{c}\text { Net } \\
\text { returns }\end{array}$} & \multirow{2}{*}{$\begin{array}{c}\text { B:C } \\
\text { Ratio } \\
0.90\end{array}$} \\
\hline $\mathbf{T}_{1}$ & White plastic mulch & & & & & & & \\
\hline $\mathbf{T}_{2}$ & Black plastic mulch & 20.27 & 6.07 & 42.78 & 25.80 & 304080 & 167612 & 1.19 \\
\hline $\mathbf{T}_{3}$ & Straw mulching $5 \mathrm{t} / \mathrm{ha}$ at $5 \mathrm{DAP}$ & 22.96 & 8.76 & 61.73 & 15.95 & 344435 & 254241 & 2.75 \\
\hline $\mathbf{T}_{4}$ & $\begin{array}{l}\text { One HW at } 20 \mathrm{DAP}+\text { Straw mulching } 5 \\
\text { t/ha at } 25 \mathrm{DAP}\end{array}$ & 25.39 & 11.19 & 78.83 & 7.06 & 380853 & 286549 & 2.94 \\
\hline $\mathbf{T}_{5}$ & Two hand weeding at $20 \& 40 \mathrm{DAP}$ & 27.32 & 13.12 & 92.42 & - & 409804 & 318346 & 3.40 \\
\hline $\mathbf{T}_{6}$ & One hand hoeing at 20 DAP & 16.53 & 2.33 & 16.40 & 39.51 & 247890 & 163148 & 1.87 \\
\hline $\mathbf{T}_{7}$ & $\begin{array}{l}\text { Hoeing at } 20 \mathrm{DAP}+\text { hand weeding at } 40 \\
\text { DAP }\end{array}$ & 17.76 & 3.56 & 25.11 & 34.98 & 266455 & 177603 & 1.92 \\
\hline $\mathbf{T}_{8}$ & $\begin{array}{l}\text { Recommended herbicide (metribuzin } 0.5 \\
\mathrm{~kg} / \mathrm{ha} \text { ) }\end{array}$ & 21.89 & 7.69 & 54.20 & 19.86 & 328413 & 244043 & 2.84 \\
\hline $\mathbf{T}_{9}$ & $\begin{array}{l}\text { Recommended herbicide }+ \text { one } \mathrm{HW} \text { at } 40 \\
\text { DAP }\end{array}$ & 22.39 & 8.19 & 57.72 & 18.03 & 335900 & 248533 & 2.78 \\
\hline \multirow[t]{4}{*}{$\mathbf{T}_{10}$} & Weedy check & 14.20 & & & 48.03 & 212972 & 129600 & 1.49 \\
\hline & $\operatorname{SEm}( \pm)$ & 1.02 & & & & 15305 & 24726.99 & 0.216 \\
\hline & CD at $5 \%$ & 2.99 & & & & 44804 & 72386.35 & 0.632 \\
\hline & CV\% & 8.52 & & & & 8.52 & 20.15 & 16.918 \\
\hline
\end{tabular}


Fig.1 The images of the experimental site at RVSKVV, Gwalior M.P. India. 30 days crop (Left) and 60 days crop (Right)
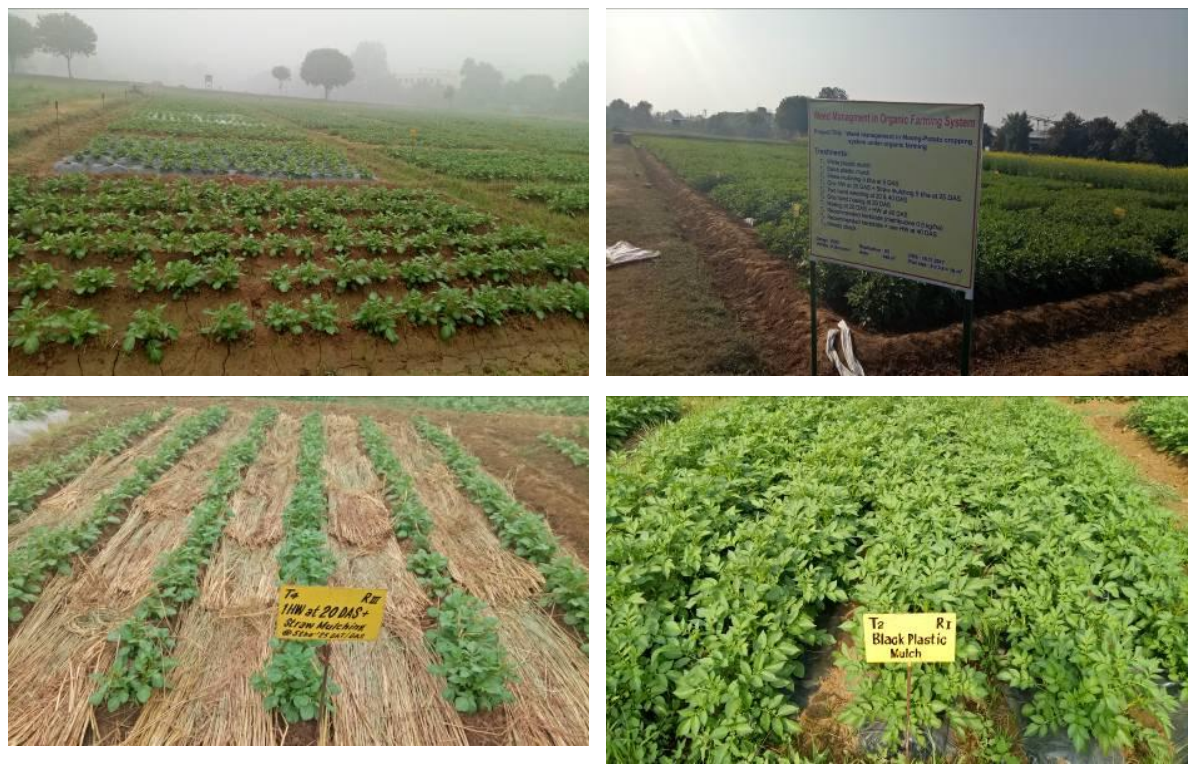

Fig.2 Weekly mean weather conditions of maximum and minimum temperature, humidity, evaporation and rainfall in 2016-7 and 2017-18 during the potato crop season

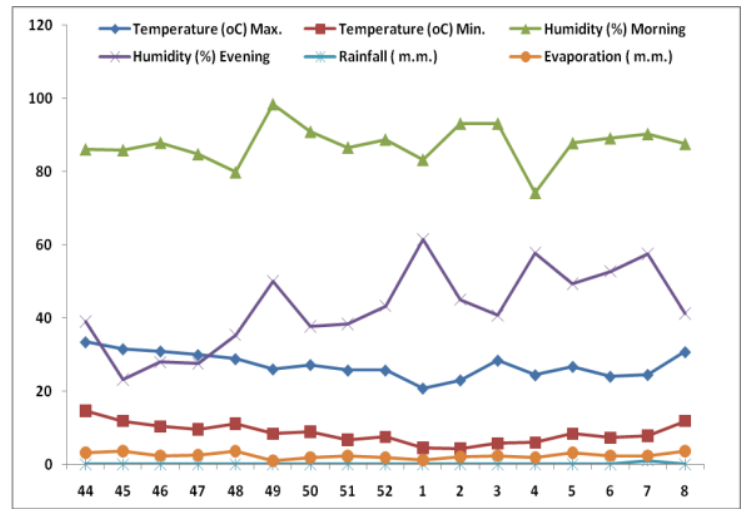

In conclusion, although inclusion of legume crops in a crop ration improve the soil health in terms of microbial properties of soil with organic nutrition. Two hand weeding at 20 and 40 DAP resulted in better control of weeds with $81.70 \%$ efficiency of weed control and maximum tuber yield (27.32 t/ha) followed by one hand weeding at 20 DAP with straw mulch application 5t/ha at 25 DAP (25.39 t/ha) and also fetched highest net returns \& $\mathrm{B}: \mathrm{C}$ ratio (Table 4 ). The non-

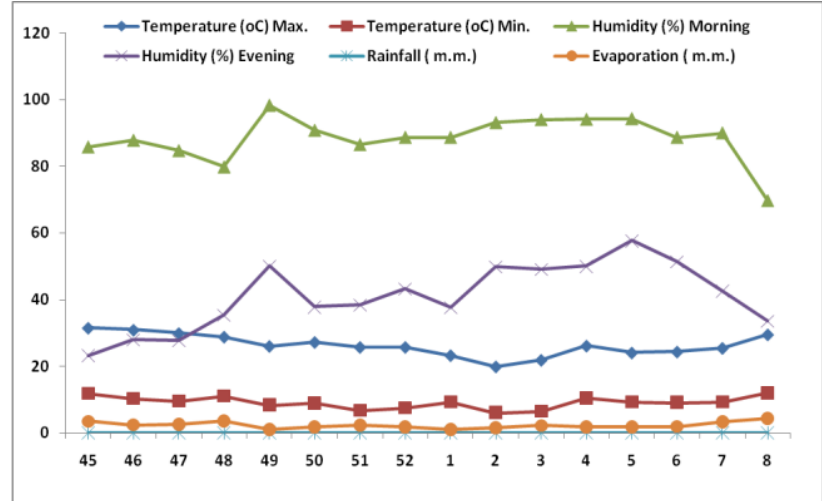

chemical methods of weed control Cultural operations and mulches has a significant effect on potato weed density so that the least amount of weed density was obtained by application of straw mulch. It is also concluded that non-chemical treatments can control weeds as much as herbicides. Use of mulches prevents the germination, growth and development of weeds, both in the initial and subsequent stages of weed growth. 
So it can be concluded that non-chemical methods of weed control can be more costly than the use of chemicals due to labour and cost related to mulch preparation but ecological methods such as application of plant residues can be more environmental friendly. The use of chemicals /herbicides is the harmful for health of humans, animals and living organisms present in the soil.

\section{References}

Ahuja, K.N., Yaduraju N.T., Singh, R. and Singh. D.K. 1999. Chemical weed control in potato (Solanum tuberosum L.). Indian Journal Weed Sciences 31:8-12.

Azadbakht, A., Alebrahim, M. T., Mohammadduost Chamanabad, H. R., Ghavidel, A., Karbalaei Khiavi, H. (2017): The effect of chemical and non-chemical weed management methods on chemical and biological properties of soil in potato cultivation in Ardabil province. Iran. - Applied Ecology and Environmental Research 15(3): 771-783.

Dejam, M., Ebadi Pour, A. R., Pourazar, R., Muhtasebi, M. (2010): Effect of mechanical (weeding) and chemical control on yield of onion cultivars. Journal of weeds ecology 1: 31-40.

Dubey, S.K. and Agrawal, S., 1999. Effect of phosphate -solubilizing microorganisms as single and composite inoculants on rainfed soybean in Vertisol. Indian Journal of Agricultural Sciences. 69 (8):611-613.

Hutchinson, P., Beutler, B., Farr, J. (2011). Hairy nightshade (Solanum sarrachoides) competition with two potato varieties. - Weed Science 59: $37-42$.

Jafari, R., Veisanlo, F., Javan, R. (2013): Weeds associated with potato (Solanum tuberosum) crops. -
International Journal of Agriculture and Crop Sciences 6 (20): 1403-1406.

Khalak, A. and A.S. Kumaraswany, 1993. Weed biomass in relation to irrigation and mulching and economics of mulching potato crop under conditions of acute water scarcity. J. Indian Potato Assoc., 20: 185-189.

Kunz, C., Weber, J. F., Gerhards, R. (2015): Benefits of Precision Farming Technologies for Mechanical Weed Control in Soybean and Sugar BeetComparison of Precision Hoeing with Conventional Mechanical Weed Control. - Agronomy 5(2): 130-142.

Lal, S.S. and Gupta, A. 1984. Efficacy of different herbicides for controlling weeds in potato. Annual Conf Indian Soc. Weed Sci., BHU, Varanasi, pp. 36.

M. Masud Mahmood, Khalid Farooq, Amjad Hussain and Raham Sher, (2002). Effect of Mulching on Growth and Yield of Potato Crop. Asian Journal of Plant Sciences, 1: 132-133.

Majd, R., Mohammaddust Chamanabad, H R., Alebrahim, M. T., Nateghi, G. A. (2014): The evaluation of solarization efficacy with polyethylene sheet mixed with chemical and physical treatments for weeds control and potato (Solanum tuberosum) yield.Research In Crop Ecosystem 1(2): 3343.

Mohammadduost, Chamanabad, H. R., Asghari1, A., Habibi, G. h. R., Pourmorad K. B. (2011): Effect of herbicides and crop residue on integrated weed control in potato. Electronic Journal of Crop Production 4(1): 171-185.

Momirovic, N.M., M.M. Misovic, Z.A. Brocic, S. Jevtic and B. Lazic, (1997). Effect of organic mulch application on the yield of potato 
seed crop. Acta Hortic., 462: 291296.

Monteiro, A., Henriques, I. and Moreira. I. 2011. Critical period for weed control in potatoes in the Huambo Province (Angola). Planta Daninha 29:351-362.

Najafi, H., Hasanzadeh, M., Rashaed Mohasel, M. H., Zand, E., Baghestani, M. A. (2016): Ecological management of agricultural weeds. - Iranian Plant Protection Research Institute, 559565.

Pandey, A.K. 2000. Weed management in vegetable crop, p. 132-138. Proc. Natl. Training Course on Vegetable Production. Indian Institute of Vegetable Research, Varanasi, India.

Raymond V. Barbhen N, Zhong Chen W, David N. Karowe $\mathrm{Z}$ and Angela Spickard (2004). $C_{3}$ grasses have higher nutritional quality than $\mathrm{C}_{4}$ grasses under ambient and elevated atmospheric $\mathrm{CO}_{2}$. Global Change Biology (2004) 10, 1565-1575, DOI: 10.1111/j.1365-2486.2004.00833.x

Ruiz, J.M., J. Hernandez, N. Castilla and L. Romero, 1999. Potato performance in response to different mulches. J. Agric. Food Chemistry, 47: 2660-2665.

Sand, G.S. and N.T. Singh, (1974). Effect of organic mulches on the hydrothermal regime of soil and growth of potato crop in Northern. Indian Plant Soil, 40: 35-47.

Saini, V.K., Bhadari, S.C., Sharma, S.K. and Tarafdar, J.C., (2005). Assessment of microbial biomass under integrated nutrient management in soybean- winter maize cropping sequence. Journal of the Indian Society of Soil Sciences. 53(3): 346-35.

Singh, V.P. and Bhan, V.M. 1999. Herbicidal control of weeds in potato (Solanum tuberosum L.) in Vertisol. Indian J. Weed Sci. 31:214-217.

Sood M.C. and R.C. Sharma, (1985). Effect of pine needle mulch on tuber yield and fertilizer economy of potato in Shimla hill soil. Journal of the Indian Society of Soil Sciences, 33: 141-144.

Wall, D.A. and Friesen, D.H. 1990. Effect of duration of green foxtail (Setaria viridis) competition on potato (Solanum tuberosum L.) yield. Weed Technol. 4:539-542.

Weber, J. F., Kunz, C. H., Gerhards, R. (2016): Chemical and mechanical weed control in soybean (Glycine max). - University of Hohenheim, Institute of Phytomedicine, OttoSander-Strabe 5, 70599 Stuttgart, Germany, 171-176.

Zaki, M. M., Saleh, E. A., Mohamed Sonya, H., Rahal, A., Sadik, A. S. (2014): Persistence of Sencor herbicide in Streptomycetes-inoculated soil and its effect on some microbial soil. - Int. J. Curr. Microbiology App. Science 3(3): 726-738.

Zand, E., Baghestani, M. A., Bitarafan, A., Shimi, P. (2007): A guidelines for herbicides in Iran. - Jihad Daneshgahi Mashhad publications.

\section{How to cite this article:}

Varsha Gupta, Ekta Joshi, Deep Singh Sasode Lakhan Singh, B.S. Kasana and Singh, Y.K. 2019. The Effect of Chemical and Non-Chemical Control Methods on Weeds and Yield in Potato (Solanum tuberosum L.) Cultivation under Potato Based Organic Cropping System. Int.J.Curr.Microbiol.App.Sci. 8(07): 2737-2747. doi: https://doi.org/10.20546/ijcmas.2019.807.342 